\title{
BP1, a potential biomarker for breast cancer prognosis
}

\author{
Yaoxian Lou1, Yassi Fallah², Kellie Yamane ${ }^{3}$ \& Patricia E Berg*,1 \\ ${ }^{1}$ Department of Biochemistry \& Molecular Medicine, George Washington University, Washington, DC 20037, USA \\ ${ }^{2}$ Department of Oncology, Georgetown University Medical Center, Lombardi Comprehensive Cancer Center, Washington, DC \\ 20057, USA \\ ${ }^{3}$ NantOmics, Diagnostic Center in Montgomery County, Rockville, MD 20850, USA \\ *Author for correspondence: Tel.: +1 202994 2810; peb@gwu.edu
}

Homeobox genes are critical in tumor development. An isoform protein of DLX4 called BP1 is expressed in $80 \%$ of invasive ductal breast carcinomas. BP1 overexpression is implicated in an aggressive phenotype and poor prognosis. BP1 upregulation is associated with estrogen receptor negativity so those tumors do not respond to antiestrogens. Breast cancer is the second leading cause of death in women. BP1 could serve as both a novel prognostic biomarker for breast cancer and a therapeutic target. In this review, we address the role of BP1 protein in tumorigenesis of breast cancer and four other malignancies. A number of functions of BP1 in cancer are also discussed.

First draft submitted: 28 June 2017; Accepted for publication: 31 January 2018; Published online: 26 March 2018

Keywords: biomarker $\bullet$ BP1 $\bullet$ BP1 target $\bullet$ breast cancer $\bullet$ distal-less family $\bullet$ DLX4 $\bullet$ estrogen receptor $\bullet$ homeobox genes $\bullet$ isoform $\bullet$ oncogene

Female breast cancer represents $14.6 \%$ of all new cancer cases in the USA according to the National Cancer Institute [1]. An estimated 252,710 new cases of invasive breast cancer are expected to be diagnosed in women in the USA in 2017. It is also estimated that $30 \%$ of newly diagnosed cancers in women will be breast cancers [2].

Breast cancer is a hormone-dependent cancer, and estrogen (17 $\beta$-estradiol) plays a critical role in the initiation and the progression of this disease [3]. Estrogen, produced by the ovaries, affects the growth and function of mammary glands by binding to the estrogen receptors (ERs) $\alpha$ and $\beta$. Through dimerization of the receptor, translocation to the nucleus and interaction with estrogen response elements in the promoter region of targeted genes, gene expression leads to multiple biological outcomes [4]. $70 \%$ of invasive breast cancers are ER positive $\left(\mathrm{ER}^{+}\right)$, making antiestrogens essential in treating these patients [5]. Targeting the ER or its pathway using tamoxifen (a selective ER modulator) or fulvestrant (an ER downregulator) have been successful therapeutic strategies [6]. However, drug resistance remains a major challenge in treating breast cancer. The main pathway leading to resistance involves loss of ER expression or selection of an ER-negative population of cells. Moreover, these ER-negative breast cancers have a higher histologic grade and a higher proliferative rate and are associated with poorer prognosis. This highlights the key importance of finding alternative targets to treat these patients and identifying a new biomarker for breast cancer prognosis.

BP1 protein, an isoform of $D L X 4$, belongs to the homeobox gene family which includes regulatory genes implicated in early development and cell differentiation that are frequently dysregulated in cancer [7]. Therefore, targeting BP1 may provide a new avenue for breast cancer management. The first related paper was published in 1998, when the authors mapped this new homeobox gene to chromosome 17q21 and characterized its role in repression of the $\beta$-globin gene [8]. During the last 20 years, several studies have been carried out to measure BP1 levels in breast cancer and other carcinomas, with the primary aim of confirming its diagnostic and prognostic value as a biomarker. We are writing this review to consolidate and update all of the available information on BP1.

\section{Homeobox genes}

Many genes involved in normal embryonic development contribute to tumorigenesis. The transformation of a healthy cell to a neoplastic cell requires activation and inhibition of a very tightly regulated system of genes

Future $\because$ Medicine 
including tumor suppressors and oncogenes. Transcription factors regulate many of these processes. A group of transcription factors involved in the control of development and differentiation of many organisms contains a homeodomain. This is a highly conserved region of 60 amino acids that is encoded by a 180 base pair DNA sequence, the homeobox. Homeobox genes play key roles in both morphogenesis and oncogenesis. They are evolutionarily highly conserved and encode homeoproteins that have major impact on their target genes. The role of homeobox genes was first documented in the embryogenesis of Drosophila melanogaster [9]. In the 1990s, abnormalities in D. melanogaster mutants were documented and homeobox genes were later confirmed in humans [10,11]. The first evidence of specific domains in these genes was characterized by nuclear magnetic resonance $[12,13]$.

Multiple studies have demonstrated different expression patterns of HOX genes in malignant tissues compared with nonmalignant tissues. There are two classes of homeobox genes in vertebrates: HOX genes, which are organized into four clusters (HOX A, B, C and D) with a total of 39 members [14], and divergent genes such as IRX, MSX, $P A X$ and $D L X$ [15,16]. Studies have shown that alterations in homeobox genes can induce a variety of tumors including tumors that result in breast cancer. In humans, downregulation of HOXA5 gene expression results in loss of expression of 'guardian of the genome' tumor suppressor p 53 [17]. In breast tissue, accumulating data suggests that dysregulated $H O X$ genes are associated with malignancy, tumor development and invasion [18-20]. Overexpression of specific $H O X$ genes such as $H O X D 3$ is associated with poor prognosis, while overexpression of $H O X B 13$ is associated with invasiveness by upregulation of IL6 and downregulation of ER $\alpha$ [21,22]. HOXB7, another homeobox gene involved in mammary gland development and hematopoietic differentiation, has been reported to show increased expression in both primary breast cancer as well as distant metastases [23]. In immunodeficient mice, $H O X B 7$ overexpression promotes proliferation leading to angiogenesis [24]. In $\mathrm{ER}^{+}$breast cancer cell lines, $H O X B 7$ overexpression confers tamoxifen resistance by cross talk with the EGFR signaling pathway, suggesting that $H O X B 7$ could be a regulator of the estrogen independent breast cancer cell phenotype. Targeting this gene may improve response to tamoxifen [25].

\section{The DLX4 gene \& BP1 protein}

The Distal-less homeobox gene $D L X 4$, which is a member of the homeobox gene family, is implicated in early development and differentiation and is frequently dysregulated in neoplasms. The DLX4 gene was first isolated from a human cDNA placenta library and is located at $17 \mathrm{q} 21.33$; it is about $5761 \mathrm{bp}$ in length [26]. BP1 is an isoform of $D L X 4$ due to alternative mRNA splicing. When it was found that BP1 is a repressor of the $\beta$-globin gene and identifying BP1, DLX7 and one unidentified DLX4 protein as isoforms, to prevent confusion in the literature, the gene was given a single name, $D L X 4$, based on the fact that the $D L X 4$ DNA sequence was published first. Thus, the DLX4 gene encodes three functionally different proteins which are isoforms: BP1, DLX7 and unidentified DLX4 [27]. The National Center for Biotechnology Information (NCBI) database is somewhat confusing in this regard - the gene is called $D L X 4$, but $B P 1$ is named $D L X 4$ gene variant 1 and $D L X 7$ is called $D L X 4$ gene variant 2. While, there are three isoforms in the DLX4 protein (Q92988) entry in UniPro database (UniProt release 2017_01): BP1 protein is named Isoform-1 (Q92988-1), DLX7 is called Isoform-2 (Q92988-2) and Isoform-3 (Q92988-3) corresponds to DLX4 protein (Figure 1).

BP1 protein maps to chromosome $17 \mathrm{q} 21-22$, which is a region of the DNA that is amplified in many breast cancers. This 240 amino acid protein is calculated to have a molecular weight of $26 \mathrm{kDa}$ but it is often observed at about $36 \mathrm{kDa}$ on western blots, which has been suggested to be due to post-translational modifications [27]. BP1 protein has a 116 amino acid N-terminal domain, a 64 amino acid C-terminal domain and a 60 amino acid homeobox domain. In studies of the regulation of hematopoiesis, it was found that BP1 activation is developmentally restricted and also tissue specific. It is normally expressed in adult kidney and placenta, and in low levels in fetal liver [28]. BP1 protein is a naturally occurring repressor of the human $\beta$-globin gene. It binds to DNA sequences upstream of the adult $\beta$-globin gene, inhibiting its expression during embryonic and fetal hemoglobin expression. During the switch to adult hemoglobin expression, BP1 activity is turned off [29].

In this systematic review, all the studies carried out so far on BP1 expression in different cancers, including breast carcinoma, leukemia, prostate, lung and ovarian cancer, have been considered and reported. In additon, a number of functions of BP1 in cancer have been also addressed. 


$\begin{array}{llr}\text { Q92988 } & \text { DLX4_HUMAN } \\ \text { Q92988-2 } & \text { DLX4_HUMAN } & \\ \text { Q92988-3 } & \text { DLX4_HUMAN } & \\ & & \\ \text { Q92988 } & \text { DLX4_HUMAN } & \\ \text { Q92988-2 } & \text { DLX4_HUMAN } & \\ \text { Q92988-3 } & \text { DLX4_HUMAN } & \\ & & 11 \\ \text { Q92988 } & \text { DLX4_HUMAN } & 4 \\ \text { Q92988-2 } & \text { DLX4_HUMAN } & 46 \\ \text { Q92988-3 } & \text { DLX4_HUMAN } & \\ & & 168 \\ \text { Q92988 } & \text { DLX4_HUMAN } & 96 \\ \text { Q92988-2 } & \text { DLX4_HUMAN } & 10 \\ \text { Q92988-3 } & \text { DLX4_HUMAN } & \\ & & \\ & & 11 \\ \text { Q92988 } & \text { DLX4_HUMAN } & \\ \text { Q92988-2 } & \text { DLX4_HUMAN } & \\ \text { Q92988-3 } & \text { DLX4_HUMAN } & \end{array}$

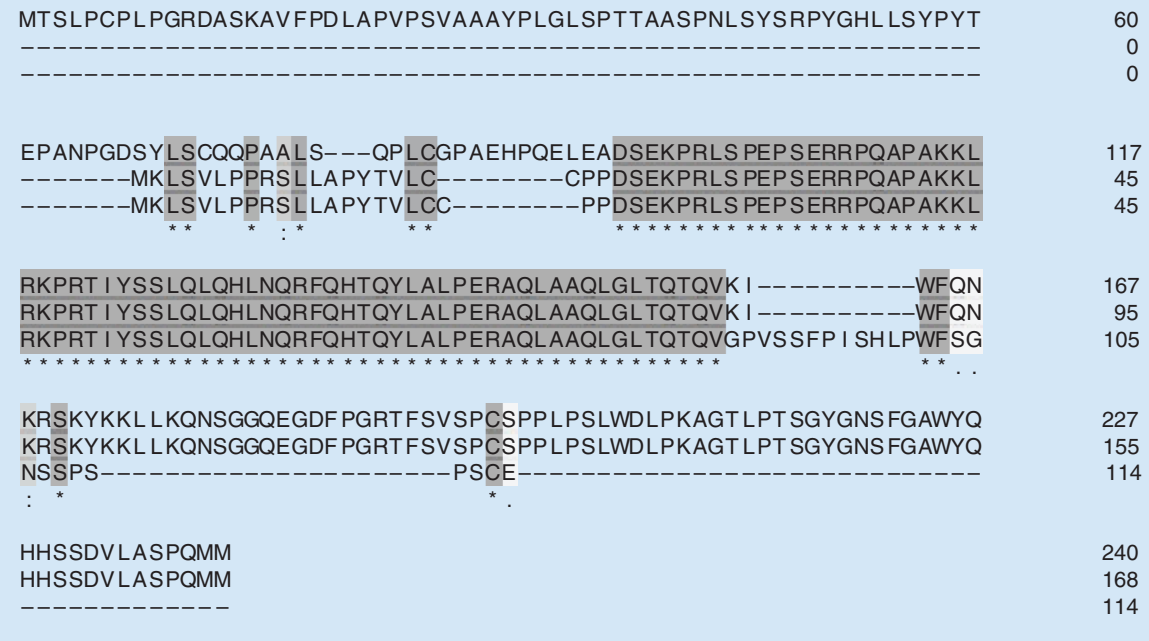

Figure 1. Amino acid sequence alignment between BP1 (Q92988-1), DLX7 (Q92988-2) and an unidentified isoform protein of DLX4 (Q92988-3) in UniPro database (UniProt release 2017_01). The sequences were obtained from the UniProt database which has incorrectly identified the BP1(Q92988-1) sequence as DLX4 protein (Q92988) in the alignment result.

\section{BP1 in breast cancer}

\section{Breast cancer}

Breast cancer remains the second leading cause of death in women. Each year, over 1 million new cases of breast cancer are diagnosed worldwide [30]. Breast cancer is a heterogeneous disease, and successful treatment for patients is dependent on a specific and selective panel of biomarkers. Breast-conserving surgery, mastectomy and in some cases radiation therapy are common treatment options for localized breast cancer. To improve prognosis, adjuvant therapies, which include chemotherapy, targeted therapy and antihormone therapy, are used. Hormone receptor status by immunohistochemical staining directs the regimen of therapies given by oncologists. However, drug resistance remains a major challenge in treating breast cancer. It is known that in about 30\% of cases, antiestrogen resistance occurs with cells still expressing ERs [31]. Moreover, when cancer cells become resistant to antiestrogens and depend on growth factors or alternative pathways for survival, finding other methods to successfully block growth and stunt survival of this population of cancer cells is essential. In addition, clinical studies have demonstrated that racial disparities may exist in many cancers, especially in breast cancer and prostate cancer among African-American and Caucasian populations. Generally, both African-American women and men have more aggressive cancers and do not respond well to therapy [32]. Researchers have been trying to find alternative targets to treat these patients. Some studies have addressed the issue of the possible role of BP1 as a potential new target.

\section{Expression}

In earlier study in which semiquantitative reverse transcription polymerase chain reaction (RT-PCR) was performed to evaluate BP1 expression, BP1 was active in $80 \%$ of invasive ductal breast tumors, where $100 \%$ of ER-negative tumors were BP1 positive, compared with $73 \%$ of ER-positive tumors ( $\mathrm{p}=0.03$ ) [33]. BP1 expression may also be associated with race: $89 \%$ of the tumors of African-American women expressed BP1 while $57 \%$ of Caucasian women showed BP1 positivity $(\mathrm{p}=0.04)$. Moreover, the frequent expression of BP1 in all tumor grades suggests that activation of BP1 is an early event. These are important findings that have both scientific and clinical implications since ER-negative tumors are more aggressive, with poor prognosis making them challenging to treat in the clinic [34].

A study by $\mathrm{Yu}$ et al. in China investigated BP1 mRNA expression in 82 breast cancer tissues, 12 near-adjacent tissues and ten far-adjacent tissues using RT-PCR [35]. The expression rates of BP1 in near and far adjacent tissues were 16.67 and $0 \%$, respectively, while the expression rate in the breast cancer group was 64.63\%. Note that BP1 expression was $57 \%$ in Caucasian women in the study cited above [34]. However, those authors failed to demonstrate the correlation between BP1 expression and ER status only based on the analysis of the frequency of BP1 mRNA expression in different tumor samples. In this regard, to further clarify the role of BP1 in breast cancer, 

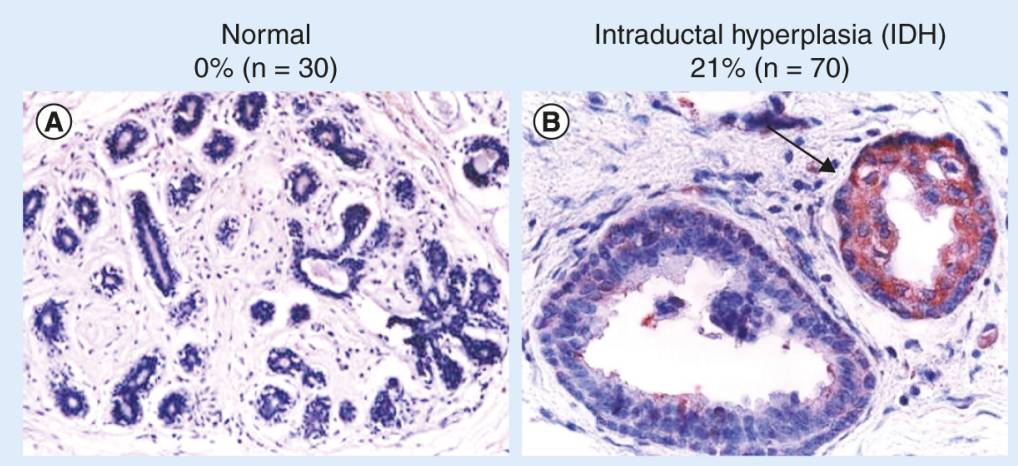

Ductal carcinoma in situ (DCIS) $46 \%(n=100)$

Invasive ductal carcinoma (IDC) $81 \%(n=100)$



Figure 2. BP1 positivity increases with progression (normal $\rightarrow$ intraductal hyperplasia $\rightarrow$ ductal carcinoma in situ $\rightarrow$ invasive ductal carcinoma) in immunostaining . (A) Normal reduction mammoplasty. $100 \times$. (B) Introductal hyperplasia (IDH). The arrow identifies duct with BP1 expression (red color indicates BP1 staining). 200×. (C) Ductal carcinoma in situ (DCIS). Expression of BP1 in DCIS is heterogeneous. Cells in on duct are homogeneously immunoreactive, while the other two ducts contain many BP1 negative cells (arrow). 150×. (D) Invasive ductal carcinoma. The infiltrating cancer cells are stongly BP1 positive (red color), while the normal (N), typical intraductal hyperplasia (IDH), and atypical intraductal hyperplasia (AIDH) are either negative or weakly positive. 100×. Reprinted with permission from [38] ( Springer Nature (2015).

For color figures please see online at https://www.futuremedicine.com/doi/full/10.2217/bmm-2017-0212

they quantitated BP1 mRNA in 142 Chinese women's invasive breast tumors and demonstrated an association between high BP1 mRNA expression, lymph node metastasis and high histological grade in the recent research. This study confirmed the data that ER-negative breast tumors express higher levels of BP1 mRNA, but no significant association between BP1 expression and HER2 status was identified [36].

Inflammatory breast cancer (IBC) is an aggressive subtype of breast cancers that has proven to be very difficult to treat. Paraffin-embedded tissue sections from 45 cases of IBC (nine with paired metastatic lymph nodes) and different controls were assayed immunohistochemically for BP1 expression. 100\% of the tested samples were BP1-positive which suggests that BP1 may play a role in the aggressiveness of these patients' breast cancers [37].

\section{Progression \& metastasis}

BP1 levels increase with the progression of tumor development: BP1 protein expression is detected in $0 \%$ of normal adult breast tissue, $21 \%$ of ductal hyperplasia, $46 \%$ of ductal carcinoma in situ and $81 \%$ invasive ductal carcinoma $(p<0.0001)$ in BP1 immunostaining (Figure 2) [38]. This suggests BP1 protein expression may reliably reflect or directly contribute to tumor progression and/or invasion. In this research, using a proliferation marker, Ki67, Man et al. also demonstrated that BP1 positivity correlates with increased proliferation when BP1 positive cells were compared with BP1 negative cells. In addition, in ER-negative Hs578T cells, overexpression of BP1 increased the cell proliferation rate by two- to fivefold $(\mathrm{p}<0.005)$, and enhanced the in vitro invasive activity by 25 - to 65 -fold ( $<$ 0.001) [39]. In a mouse model, it has recently shown that BP1 overexpression in MCF-7 breast cancer cells leads to increased proliferation, increased tumor size and estrogen-independent tumor formation [40].

BP1 has also been implicated in metastasis. Among 46 cases of IBC, BP1 was shown to be positive in the lymph nodes of all nine cases which had metastasized [37]. In addition, Yu et al. observed that invasive ductal breast 
Table 1. Selected studies on expression of BP1 in other neoplasms.

\begin{tabular}{|c|c|c|}
\hline Type of cancer & BP1-positive expression data & Ref. \\
\hline Leukemia & $\begin{array}{l}81 \% \text { of the pediatric and } 47 \% \text { of the adult cases ( } 39 \text { AML patients, including } 18 \text { pediatric under the age of } 18 \text { and } 21 \text {; } \\
\text { adult in } 18 \text { years of age or older) were BP1 positive }\end{array}$ & [44] \\
\hline Prostate cancer & $70 \%$ of cases (50 tissue sections, 123 tissue microarray platforms) were BP1 positive & [45] \\
\hline Lung cancer & $\begin{array}{l}78 \% \text { of lung cancer tissues and } 13 \% \text { adjacent tissues but } 0 \% \text { of the normal tissues ( } 46 \text { lung cancer tissues, } 46 \text { adjacent } \\
\text { tissues, } 46 \text { noncancer lung tissues from } 46 \text { lung cancer patients) were BP1 positive }\end{array}$ & [46] \\
\hline Ovarian cancer & $\begin{array}{l}47 \% \text { ( } 32 \text { ovarian carcinoma cases, including } 13 \text { low-grade ovarian carcinomas and } 19 \text { high-grade ovarian carcinomas) } \\
\text { were BP1 positive }\end{array}$ & [47] \\
\hline
\end{tabular}

tumors with a high BP1 mRNA level are significantly associated with positive lymph node metastasis. In this study, according to Kaplan-Meier curves, women with grade III tumors overexpressing BP1 showed decreased survival compared with those women with grade III tumors expressing low BP1, demonstrating that the intensity of BP1 levels is associated with survival [36]. Song et al. reported that there was a high percentage of cyclin D1 and BP1 co-expression in 86 cases of breast cancer tissues [41]. BP1 may be capable of acting with cell cycle modulators to increase tumor growth while preventing cells from undergoing apoptosis, regarded as a direct cause of malignancy.

\section{Mechanism of BP1 activation}

Approximately $33 \%$ of metastatic breast tumors examined by Cavalli et al. demonstrated increased BP1 copy number with a frequency of 36 and $29 \%$ in primary breast tumors and sentinel lymph node metastasis, respectively [42]. This amplification was associated with BP1 protein positivity by immunostaining in all cases. This suggests that BP1 can be activated by DNA amplification. Further study shows that increased copy number of the $B P 1$ gene in breast cancer is not a result of co-amplification associated with HER2/NEU, which is the main amplification driver on the $17 \mathrm{q}$ region $[43]$.

\section{BP1 in other neoplasms}

It is important to also understand the expression and the role of BP1 in other cancers. Several studies have reported BP1 activation in leukemia, prostate cancer, lung cancer and ovarian cancer (see Table 1).

\section{Leukemia}

About 62,130 new cases of all kinds of leukemia and 24,500 deaths are estimated in 2017 [48]. Many studies have investigated driver genes in different types of leukemia. Using semiquantitative RT-PCR to investigate the role of BP1, it found that BP1 was highly expressed in $63 \%$ of bone marrows from patients with acute myeloid leukemia, including $81 \%$ of the pediatric and $47 \%$ of the adult cases, and in $32 \%$ of pediatric T-cell acute lymphocytic leukemia (ALL) cases, but was not found in any of the pre-B ALL cases [44]. The same study demonstrated that coexpression of BP1, DLX7 and DLX4 occurred in a significant number of the same cases. A group in China detected the expression of BP1 mRNA in 70 adult acute leukemia cases, discovering that the BP1 was highly expressed in $57 \%$ of acute myeloid leukemia patients but could not be detected in adult ALL [49].

Moreover, we compared the growth-inhibitory and cyto-differentiating activities of all-trans retinoic acid (ATRA) in NB4 (ATRA-responsive) and R4 (ATRA-resistant) acute promyelocytic leukemia cells relative to BP1 levels [50]. NB4 and R4 cells both expressed BP1, while this expression was repressed after ATRA treatment of NB4 cells but not R4 cells. In NB4 cells engineered to overexpress BP1, proliferation was no longer inhibited and differentiation was reduced two- to threefold. Clinically, BP1 levels were increased in all pretreatment acute promyelocytic leukemia patients tested, while BP1 expression was decreased in $91 \%$ of patients after combined ATRA and chemotherapy treatment. In a follow-up study, two patients relapsed during follow-up and one patient exhibited a 42-fold increase in BP1 expression, while the other showed no change. This suggests that BP1 may play a role in resistance to therapy.

\section{Prostate cancer}

Prostate cancer, the most common cancer in men, is a hormone-dependent solid tumor. Although prostate specific antigen has been a major marker to detect progression of prostate cancer, accumulating clinical data have shown that 
false positive and false negative results are common. This led to researchers investigating other potential markers to detect prostate cancer at its early stage.

Prostate cancer was examined for activation of BP1 [45]. In this study, significant BP1 immunoreactivity was identified in approximately $70 \%$ of prostatic tumors, whether the analysis was performed on tissue sections ( 50 cases) or tissue microarray platforms (123 cases). Consistent with the BP1 data on breast cancer, malignant prostate tissues showed higher expression of BP1 compared with hyperplastic and normal tissues. In tissue sections, 12 cases with paired carcinoma and prostatic intraepithelial neoplasia showed agreement, both components exhibiting strong immunoreactivity. Tumor proliferation, assayed with Ki-67 (MIB-1) immunostaining, was higher in cancer cells that were BP1 positive relative to those that were BP1 negative, in agreement with the data in breast cancer cells. Thus, BP1 may directly contribute to tumor progression and metastasis, and importantly, BP1 could become a potential new biomarker for prognosis of prostate cancer.

\section{Lung cancer}

According to the American Cancer Society, in 2017, about 222,500 new cases of lung cancer will be diagnosed and about 155,870 deaths will be from lung cancer [48]. Understanding the pathogenesis of this disease is essential since $14 \%$ of all cancers are lung cancers. Prognostic biomarkers are essential for early diagnosis of lung cancer. One early study in China reported that $78 \%$ of lung cancer tissues and $13 \%$ adjacent tissues but none of the normal tissues were found to have $B P 1$ gene overexpression, showing a significant difference in BP1 expression between the tissues ( $\mathrm{p}<0.01$ ) [46]. In addition, about $80-85 \%$ of lung cancers are non-small-cell lung cancers (NSCLCs). An interesting study by Yu et al. demonstrated significant positive associations between BP1 mRNA quantitative expression and the pathological differentiation grade and tumor staging, indicating that BP1 may be a part of a molecular pathway involved in NSCLC development and/or progression [51]. Patients with high BP1 mRNA expression had a poorer prognosis in terms of both disease-free survival and overall survival rates. Additionally, BP1 mRNA expression level was an independent predictor of disease-free survival. In this regard, the BP1 mRNA level could be a novel prognostic marker for NSCLC.

\section{Ovarian cancer}

Ovarian cancer ranks fifth in cancer deaths among women, accounting for more deaths than any other cancer of the female reproductive system. The American Cancer Society estimates that about 22,440 women will receive a new diagnosis of ovarian cancer in the USA in 2017 [48]. In Hara's study, 47\% of the ovarian carcinoma cases were found to exhibit extensive BP1 expression [47]. The data showed that BP1 expression in ovarian carcinomas is strongly associated with high tumor grade and correlates with disease stage. The association of BP1 overexpression with indicators of poor prognosis is highly consistent with the finding that overexpression of BP1 in ovarian cancer cells induces levels of VEGF and FGF-2, stimulates endothelial cell growth and leads to increased microvessel density in tumors.

\section{BP1 protein functions}

Since BP1 has been shown to play a role in carcinogenesis and could be a useful new biomarker in the early detection of multiple cancers, understanding more about BP1 functions is important. Functionally, we demonstrated that $\mathrm{BP} 1$ is a repressor of the $\beta$-globin gene, while DLX7, an isoform of BP1 protein, binds to the same DNA sequence upstream of the $\beta$-globin gene but lacks the ability to repress it [52]. Thus, the functions of BP1 and DLX7 are clearly different in this context. BP1 acts to repress embryonic and fetal globin genes during early development but is itself repressed during normal adult erythropoiesis.

The transformation of a healthy cell to a neoplastic cell requires activation and inhibition of a very tightly regulated system of genes including tumor oncogenes and suppressors. As a transcription factor, BP1 protein has been shown to have effects on many of these processes (see Table 2).

c-Myc

$c-M y c$ is a regulator gene that codes for a transcription factor. The protein encoded by this gene is a multifunctional, nuclear phosphoprotein that plays a role in cell cycle progression, apoptosis and cellular transformation. Persistently expressed Myc is found in many cancers. TGF- $\beta$ and members of the TGF- $\beta$ superfamily function within the body as a signaling cascade which includes the activation of regulatory proteins that result in various downstream effects. A study by Trinh $e t a l$. found that BP1 protein blocks TGF- $\beta$-mediated repression of $c-M y c$ transcription, and also 


\begin{tabular}{|c|c|c|c|}
\hline Gene & Associated with & Relationship with BP1 & Ref. \\
\hline$C-M Y C$ & Proliferation, differentiation, apoptosis & $\begin{array}{l}\text { BP1 blocks TGF- } \beta \text {-mediated repression of } c-M y c \text { transcription and also induces } c-M y c \text { levels } \\
\text { independently of TGF- } \beta / \text { Smad signaling }\end{array}$ & [53] \\
\hline$B C L-2$ & Antiapoptotic & BP1 overexpression induces increased $\mathrm{BCl}-2$ expression & [54] \\
\hline VEGF & Angiogenesis & Overexpression of BP1 in ovarian cancer cells increases levels of VEGF & [47] \\
\hline$B R C A 1$ & Tumor suppressor, DNA damage repair & BP1 protein represses $B R C A 1$ expression & [54] \\
\hline
\end{tabular}

induces $c-M y c$ levels independently of TGF- $\beta /$ Smad signaling. Moreover, BP1 inhibits TGF- $\beta$-mediated induction of $\mathrm{p} 15^{\text {Ink } 4 \mathrm{~B}}$ and $\mathrm{p} 21^{\text {WAF1/Cip } 1}$ expression by blocking the TGF- $\beta /$ Smad signaling pathway in normal and malignant epithelial cell lines [53].

\section{$\mathrm{BCl}-2$}

$\mathrm{Bcl}-2$ has been found to regulate apoptosis by preventing the expression of antiapoptotic genes or through the expression of genes that promote apoptosis. BP1 protein binds to the regulatory region of the $\mathrm{Bcl}-2$ gene which is an antiapoptotic gene and considered to be an oncogene, resulting in elevated expression of $\mathrm{Bcl}-2$ protein and inhibition of apoptosis in MCF-7 breast cancer cells challenged with TNF- $\alpha$ [54]. Increased BP1 is associated with decreased processing and activation of caspase-7, caspase- 8 and caspase-9, and increased expression of full-length poly ADP-ribose polymerase. Thus, high BP1 expression can lead to decreased cell death, and as mentioned above, increased proliferation.

\section{Vascular endothelial growth factor}

VEGF functions normally in cells to create new blood vessels. In cancer, overexpression of $V E G F$ has been shown to enhance metastasis due to angiogenesis. Overexpression of BP1 in ovarian cancer cells induces levels of VEGF and FGF-2, stimulates endothelial cell growth and leads to increased microvessel density in tumors, but it is not known whether BP1 protein directly activates FGF-2 transcription [47].

\section{$B R C A 1$}

$B R C A 1$, which is a tumor suppressor gene, has been hypothesized to play a role in DNA damage repair, and many studies have reported a reduction in BRCA1 mRNA and protein levels following extended exposure to DNA damaging agents. BP1 protein appears to repress $B R C A 1$ expression through direct binding to its first intron. Three breast cancer cell lines engineered to overexpress BP1 showed decreased BRCA1 RNA and protein, while cells in which BP1 was knocked down by siRNA treatment showed increased BRCA1 expression, suggesting that BP1 activity may contribute to reduced-BRCA1 in some breast cancers [55].

\section{Conclusion \& future perspective}

The main goal of developing targeted cancer therapeutics is to selectively target cancer mutations. However, targeting all mutations in a tumor is not possible due to toxicity. New gene mutations occur as cancer progresses by activating oncogenes, and preclinical studies have demonstrated that cancer cell survival relies on specific oncogenes that drive the specific disease. The 'oncogene addiction' phenomenon describes a cancer cell's dependence on individual oncogenes to sustain malignancy leading to invasion and metastasis [56]. Loss of function and overexpression of homeobox genes have significant impact in cancer. However, it is not well understood how homeobox genes control specific proneoplastic characteristics in a tissue-specific manner. One possibility is that homeotic proteins usually bind to partner proteins, which may be an important factor regulating whether the homeotic protein can bind to its DNA target. Certainly, it is important to investigate the mechanisms by which dysregulated expression of certain homeobox genes occur in solid tumors.

BP1 is an isoform of DLX4 due to alternative mRNA splicing. In the last 20 years, an increasing number of studies detecting the BP1 levels in different carcinomas, especially breast cancer, have been carried out with the aim to further highlight its possible role as a diagnostic or prognostic biomarker. Except in breast cancer, there are preliminary data suggesting that addiction to BP1 may occur in some cancers, including leukemia, prostate cancer, NSCLC and ovarian cancer. Not only does BP1 expression increase with breast tumor progression but it is also involved in regulating gene expression patterns in tumors, driving them toward a more aggressive subtype. 
Exploring BP1 as an oncogenic addiction gene would help by potentially inhibiting one driver of the breast cancer malignant phenotype.

However, it remains a challenge to target homeoproteins for therapeutics since they are transcription factors, making it crucial to investigate their signaling pathways and target genes. Since BP1 is crucial for normal hematopoiesis,

\section{Executive summary}

- To decrease the mortality of breast cancer, targeting the estrogen receptor (ER) or its pathway have been successful therapeutic strategies. However, ER-negative breast cancers can exhibit drug resistance and poor prognosis. This highlights the fact that the clinical prognosis prediction of breast cancer is still unsatisfactory.

- BP1, an isoform of DLX4, was mapped to chromosome 17q21 and its role in repression of the $\beta$-globin gene was characterized in the lab in 1998. During the last 20 years, several studies have been carried out to measure BP1 levels in cancers, especially breast cancer, with the primary aim of confirming its diagnostic and prognostic value as a biomarker.

Homeobox genes

- The homeodomain is a highly conserved region of 60 amino acids that is encoded by a 180 base pair DNA sequence, the homeobox.

- There are different expression patterns of HOX genes in malignant tissues compared with normal tissues.

The $D L X 4$ gene \& BP1 protein

- The $D L X 4$ gene, which is a member of the homeobox gene family, encodes three functionally different isoform proteins: BP1, DLX7 and an unidentified isoform protein of $D L X 4$.

- $\mathrm{BP} 1$ is derived by alternative mRNA splicing and includes 240 amino acids. In normal tissues, its activation is developmentally restricted, while in cancer BP1 can be expressed in many tissues.

- BP1 is a normal repressor of the human $\beta$-globin gene. It acts to repress embryonic and fetal globin gene expression during early development but is itself repressed during normal adult erythropoiesis.

BP1 in breast cancer

- It was found that BP1 protein is activated in $81 \%$ of invasive ductal breast tumors by immunostaining. BP1 expression shows a racial disparity: it is more frequently overexpressed in breast tumors of African-American women $(89 \%)$ than in breast tumors of Caucasian-American women $(57 \%)$.

- In inflammatory breast cancer, where women show half the survival of women with invasive ductal breast cancer, BP1 was overexpressed in $100 \%$ of the tumors, suggesting it may play a major role in aggressiveness of these types of breast tumors.

- BP1 is overexpressed in many aggressive tumor phenotypes such as triple negative breast cancer and ER-negative breast tumors.

- BP1 overexpression leads to increased cell growth, estrogen-independent tumor formation and increased proliferation. This was shown in vitro using cell lines, in experiments in which mice were injected with MCF7 cells stably overexpressing BP1 protein or control cells containing no BP1 protein and in patients with breast cancer.

- DNA amplification activates BP1. There are likely other activators yet to be discovered.

BP1 in other neoplasms

- BP1 is activated in acute myeloid leukemia and is observed with higher intensities in pediatric versus adult acute myeloid leukemia.

- Malignant prostate tissues showed higher expression of BP1 compared with hyperplastic and normal tissues.

- High BP1 mRNA levels are reported in non-small-cell lung cancer compared with normal lung tissue.

- BP1 expression in ovarian carcinoma is strongly associated with high tumor grade and correlates with disease stage.

BP1 protein functions

- BP1 acts to repress embryonic and fetal globin genes during early development but is itself repressed during normal adult erythropoiesis.

- As a transcription factor, BP1 protein has been shown to have regulatory effects on tumor oncogenes and suppressors, including C-MYC, BCL-2, VEGF and BRCA1.

Conclusion \& future perspective

- The mechanisms by which dysregulated expression of certain homeobox genes occur in solid tumors are still not well understood.

- In the last 20 years, BP1 which belongs to the homeobox gene family, has been investigated in different carcinomas with the aim to further highlight its possible role as a diagnostic or prognostic biomarker.

- Regarding the challenges of the studies of BP1, there is still significant work to be done: determining methods to better establish the prognostic ability of BP1; identifying the association between BP1 and other important molecular proteins in breast cancer; and discovering the mechanisms by which BP1 is regulated. Moreover, the molecular effects of BP1 in breast cancer as well as other malignancies could be future study directions. 
it may be necessary to target only the cancer cells and knockdown BP1 expression in those cells. It is also relevant to better investigate upstream and downstream proteins for possible therapeutic interventions. In the future, there is still significant work to be done: determining methods to better establish the prognostic ability of BP1; identifying the association between BP1 and other important molecular proteins in breast cancer; and discovering the mechanisms by which BP1 is regulated.

Future clinical studies may provide important insights on BP1 as a prognostic biomarker and potential therapeutic target in breast cancer. It is therefore of importance to thoroughly investigate the molecular effects of BP1 in breast cancer as well as other malignancies. Since accumulating data have shown that BP1 activation has a role in pathogenesis of breast cancer, downregulation of BP1 could block progression of cancer. Thus, targeting BP1 is a logical strategy to increase the survival of breast cancer patients.

\section{Financial \& competing interests disclosure}

The authors have no relevant affiliations or financial involvement with any organization or entity with a financial interest in or financial conflict with the subject matter or materials discussed in the manuscript. This includes employment, consultancies, honoraria, stock ownership or options, expert testimony, grants or patents received or pending, or royalties.

No writing assistance was utilized in the production of this manuscript.

\section{Open access}

This work is licensed under the Attribution-NonCommercial-NoDerivatives 4.0 Unported License. To view a copy of this license, visit http://creativecommons.org/licenses/by-nc-nd/4.0/

\section{References}

Papers of special note have been highlighted as: $\bullet$ of interest; $\bullet \bullet$ of considerable interest

1. National Cancer Institute. https://www.cancer.gov/

2. US breast cancer statistics. www.breastcancer.org

3. Chang M. Tamoxifen resistance in breast cancer. Biomol. Ther. 20(3), 256-267 (2012).

4. Gross JM, Yee D. How does the estrogen receptor work? Breast Cancer Res. 4(2), 62-64 (2002).

5. Shibata $\mathrm{T}$, Watari $\mathrm{K}$, Izumi $\mathrm{H}$ et al. Breast cancer resistance to antiestrogens is enhanced by increased ER degradation and ERBB2 expression. Cancer Res. 77(2), 545-556 (2017).

6. Reinert T, Barrios CH. Optimal management of hormone receptor positive metastatic breast cancer in 2016. Ther. Adv. Med. Oncol. 7(6), 304-320 (2015).

7. Abate-Shen C. Deregulated homeobox gene expression in cancer: cause or consequence? Nat. Rev. Cancer 2(10), 777-785 (2002).

8. Fu S, Strovel JW, Haga SB et al. Mapping of a new homeobox gene, BP1, near its isoform DLX7 and characterization of their roles in repression of the beta globin gene. Am. J. Hum. Genet. 63, A181 (1998).

9. Lewis EB. A gene complex controlling segmentation in Drosophila. Nature 276(5688), 565-570 (1978).

10. Kamps MP, Murre C, Sun XH, Baltimore D. A new homeobox gene contributes the DNA binding domain of the $\mathrm{t}(1 ; 19)$ translocation protein in pre-B ALL. Cell 60(4), 547-555 (1990).

11. Nourse J, Mellentin JD, Galili N et al. Chromosomal translocation $\mathrm{t}(1 ; 19)$ results in synthesis of a homeobox fusion mRNA that codes for a potential chimeric transcription factor. Cell 60(4), 535-545 (1990).

12. Billeter M, Qian Y, Otting G, Muller M, Gehring WJ, Wuthricj K. Determination of the three-dimensional structure of the Antennapedia homeodomain from Drosophila in solution by $1 \mathrm{H}$ nuclear magnetic resonance spectroscopy. J. Mol. Biol. 214(1), 183-197 (1990).

13. Qian YQ, Billeter M, Otting G, Muller M, Gehring WJ, Wuthrich K. The structure of the Antennapedia homeodomain determined by NMR spectroscopy in solution: comparison with prokaryotic repressors. Cell 59(3), 573-580 (1989).

14. Van Oostveen JW, Bijl JJ, Raaphorst FM, Walboomers JJ, Meijer CJ. The role of homeobox genes in normal hematopoiesis and hematological malignancies. Leukemia 13(11), 1675-1690 (1999).

15. Stein S, Fritsch R, Lemaire L, Kessel M. Checklist: vertebrate homeobox genes. Mech. Dev. 55(1), 91-108 (1996).

16. Popovici C, Leveugle M, Birnbaum D, Coulier F. Homeobox gene clusters and the human paralogy map. FEBS Lett. 491(3), 237-242 (2001).

17. Raman V, Martensen SA, Reisman D et al. Compromised HOXA5 function can limit $\mathrm{p} 53$ expression in human breast tumors. Nature 405(6789), 974-978 (2000).

18. Makiyama K, Hamada J, Takada M et al. Aberrant expression of HOX genes in human invasive breast carcinoma. Oncol. Rep. 13(4), 673-679 (2005). 
19. Cantile M, Pettinato G, Procino A, Feliciello I, Cindolo L, Cillo C. In vivo expression of the whole HOX gene network in human breast cancer. Eur. J. Cancer 39(2), 257-264 (2003).

20. Hur H, Lee JY, Yun HJ, Park BW, Kim MH. Analysis of HOX gene expression patterns in human breast cancer. Mol. Biotechnol. 56(1), 64-71 (2014).

21. Shaoqiang C, Yue Z, Yang L et al. Expression of HOXD3 correlates with shorter survival in patients with invasive breast cancer. Clin Exp. Metastasis 30(2), 155-163 (2013).

22. Shah N, Jin K, Cruz LA et al. HOXB13 mediates tamoxifen resistance and invasiveness in human breast cancer by suppressing ER- $\alpha$ and inducing IL-6 expression. Cancer Res. 73(17), 5449-5458 (2013).

23. Wu X, Chen H, Parker B et al. HOXB7, a homeodomain protein, is overexpressed in breast cancer and confers epithelial-mesenchymal transition. Cancer Res. 66(19), 9527-9534 (2006).

24. Carè A, Felicetti F, Meccia E et al. HOXB7: a key factor for tumor-associated angiogenic switch. Cancer Res. 61(17), 6532-6539 (2001).

25. Jin K, Kong X, Shah T et al. The HOXB7 protein renders breast cancer cells resistant to tamoxifen through activation of the EGFR pathway. Proc. Natl Acad. Sci. USA 109(8), 2736-2741 (2012).

26. Quinn LM, Johnson BV, Nicholl J, Sutherland GR, Kalionis B. Isolation and identification of homeobox genes from the human placenta including a novel member of the Distal-less family, DLX4. Gene 187(1), 55-61 (1997).

27. Berg PE, Kirolikar S. DLX4 (distal-less homeobox 4). Atlas Genet. Cytogenet. Oncol. Haematol. 15(8), 658-661 (2011).

- A short review of earlier studies about BP1.

28. Chase MB, Fu S, Haga SB et al. BP1, a homeodomain-containing isoform of DLX4, represses the beta globin gene. Mol. Cell. Biol. 22(8), 2505-2514 (2002).

- Early study showing that BP1 regulates expression of the adult $\beta$-globin gene and is important in normal erythroid differentiation.

29. Fu S, Stevenson H, Strovel J et al. Distinct functions of two isoforms of a homeobox gene, BP1 and $D L X 4$, in the regulation of the beta globin gene. Sci. Direct 278(1-2), 131-139 (2001).

30. Jemal A, Bray F, Center MM, Ferlay J, Ward E, Forman D. Global cancer statistics. CA Cancer J. Clin. 61(2), 69-90 (2011).

31. Shibata $\mathrm{T}$, Watari $\mathrm{K}$, Izumi $\mathrm{H}$ et al. Breast cancer resistance to antiestrogens is enhanced by increased ER degradation and ERBB2 expression. Cancer Res. 77(2), 545-556 (2017).

32. Carey LA, Perou CM, Livasy CA et al. Race, breast cancer subtypes and survival in the Carolina Breast Cancer Study. JAMA 295(21), 2492-2502 (2006).

33. Fu SW, Schwartz A, Stevenson $\mathrm{H}$ et al. Correlation of expression of BP1, a homeobox gene, with estrogen receptor status in breast cancer. Breast Cancer Res. 5(4), R82-R87 (2003).

-. The first study of BP1 expression in breast tumors showed that BP1 was expressed in $80 \%$ of 46 breast tumors. BP1 mRNA expression was correlated with the ability of malignant cell lines to cause breast cancer in mice.

34. Sheikh MS, Garcia M, Pujol P, Fontana JA, Rochefort H. Why are estrogen-receptor-negative breast cancers more aggressive than the estrogen-receptor-positive breast cancers? Invasion Metastasis 14(1-6), 329-336 (1994-1995).

35. Yu M, Yang Y, Niu RF et al. Clinicopathological significance of homeobox gene BP1 mRNA expression in human breast cancer. Chin. J. Cancer 23(7), 855-859 (2004).

36. Yu M, Yang Y, Shi Y et al. Expression level of beta protein $1 \mathrm{mRNA}$ in Chinese breast cancer patients: a potential molecular marker for poor prognosis. Cancer Sci. 99(1), 173-178 (2008).

37. Man YG, Schwartz A, Levine PH, Teal C, Berg PE. BP1, a putative signature marker for inflammatory breast cancer and tumor aggressiveness. Cancer Biomark. 5(1), 9-17 (2009).

38. Man YG, Fu SW, Schwartz AM et al. Expression of BP1, a novel homeobox gene, correlates with breast cancer progression and invasion. Breast Cancer Res. Treat. 90(3), 241-247 (2005).

- Investigated the relationship of BP1 expression with breast tumor progression and invasion, finding that BP1 positive tumor cells had a statistically higher proliferation rate than morphologically similar cells without BP1 expression.

39. Fu Y, Lian Y, Kim KS et al. BP1 homeoprotein enhances metastatic potential in ER-negative breast cancer. J. Cancer 1(1), 54-62 (2010).

-. Examined effects and mechanisms of BP1 action on cell proliferation and metastasis using estrogen receptor negative Hs578T cells as a model. Overexpression of BP1 increased cell proliferation by two- to fivefold and increase invasive activity by 25 - to 65-fold.

40. Fu SW, Kirolikar SP, Ginsburg E et al. Beta protein 1 homeoprotein induces cell growth and estrogen-independent tumorigenesis by binding to the estrogen receptor in breast cancer. Oncotarget 7(33), 53204-53216 (2016).

-• Investigated the effect of stable BP1 overexpression in MCF-7 breast cancer cells, finding increased proliferation and estrogen-independent tumor formation by cells overexpressing BP1.

41. Song YC, Fu SD, Wen XP, Ma ZH, Dang CX. Expression and significance of BP1 gene and cyclin D1 gene in breast cancer. Chin. J. Cancer 26(7), 709-714 (2007). 
42. Cavalli LR, Man YG, Schwartz AM et al. Amplification of the BP1 homeobox gene in breast cancer. Cancer Genet. 187(1), 19-24 (2008).

-• An important mechanism for activation of BP1 was discovered in breast cancer patients - BP1 can be actived by DNA amplification in about $33 \%$ of cases.

43. Torresan C, Oliveira MM, Pereira SR et al. Increased copy number of the DLX4 homeobox gene in breast axillary lymph node metastasis. Cancer Genet. 207(5), 177-187 (2014).

44. Haga SB, Fu S, Karp JE et al. BP1, a new homeobox gene, is frequently expressed in acute leukemias. Leukemia 14(11), 1867-1875 (2000).

45. Schwartz AM, Man YG, Rezaei MK et al. BP1, a homeoprotein, is significantly expressed in prostate adenocarcinoma and is concordant with prostatic intraepithelial neoplasia. Mod. Pathol. 22(1), 1-6 (2009).

46. Xian YS, Dang CX, Yan CX, Li HP, Fu SW, Wang ZR. Clinicopathological significance of homeobox BP1 mRNA expression in lung cancer tissue. Nan Fang Yi Ke Da Xue Xue Bao 26(8), 1173-1175 (2006).

47. Hara F, Samuel S, Liu J, Rosen D, Langley RR, Naora H. A homeobox gene related to Drosophila distal-less promotes ovarian tumorigenicity by inducing expression of vascular endothelial growth factor and fibroblast growth factor-2. Am. J. Pathol. 170(5), 1594-1606 (2007).

48. American Cancer Society. https://www.cancer.org

49. Li H, Xu KL, Pan XY, Wang HY, Li ZY. Expression of BP1 gene in adult acute leukemia. Zhonghua Xue Ye Xue Za Zhi 25(1), 38-40 (2004).

50. Awwad RT, Do K, Stevenson H et al. Overexpression of BP1, a homeobox gene, is associated with resistance to all-trans retinoic acid in acute promyelocytic leukemia cells. Ann. Hematol. 87(3), 195-203 (2008).

51. Yu M, Wan Y, Zou Q. Prognostic significance of BP1 mRNA expression level in patients with non-small-cell lung cancer. Clin. Biochem. 41(10), 824-830 (2008).

52. Fu S, Stevenson H, Strovel JW et al. Distinct functions of two isoforms of a homeobox gene, $B P 1$ and $D L X 7$, in the regulation of the $\beta$-globin gene. Gene 278(1), 131-139 (2001).

53. Trinh BQ, Barengo N, Naora H. Homeodomain protein DLX4 counteracts key transcriptional control mechanisms of the TGF- $\beta$ cytostatic program and blocks the antiproliferative effect of TGF- $\beta$. Oncogene 30(24), 2718-2729 (2011).

54. Stevenson HS, Fu SW, Pinzone JJ, Rheey J, Simmens SJ, Berg PE. BP1 transcriptionally activates bcl-2 and inhibits TNF $\alpha$-induced cell death in MCF7 breast cancer cells. Breast Cancer Res. 9(5), R60 (2007).

- First findings about the relationship of BP1 with oncogene bcl-2 in MCF7 breast cancer cells model.

55. Kluk BJ, Fu Y, Formolo TA et al. BP1, an isoform of DLX4 homeoprotein, negatively regulates BRCA1 in sporadic breast cancer. Int. J. Biol. Sci. 6(5), 513-524 (2010).

56. Weinstein IB. Cancer. Addition to oncogenes - the Achilles heel of cancer. Science 297(5578), 63-64 (2002). 
